\title{
Prevalence of Shigella species and its drug resistance pattern in Ethiopia: a systematic review and meta-analysis
}

\author{
Siraj Hussen ${ }^{1 *}$, Getamesay Mulatu ${ }^{1}$ and Zemenu Yohannes Kassa ${ }^{2}$
}

\begin{abstract}
Background: Shigella species are a major cause of dysentery and may attribute for death worldwide. Currently antibiotic resistance became the critical challenges for management of infectious disease. The aim was to conduct a systematic review and meta-analysis of Shigella species and its drug resistance pattern in Ethiopia.

Methods: A comprehensive literature search was conducted through internet searches using database of MEDLINE, PubMed, Google scholar, EMBASE, HINARI, Cochrane Library and reference lists of previous prevalence studies from January 1999 to November 2018. Results were presented in forest plot, tables and figures with 95\% Cl. The Cochrane $\mathrm{Q}$ test and $\mathrm{I}^{2}$ test statistic were used to test heterogeneity across studies. The Pooled estimate of Shigella species and its drug resistance pattern was computed by a random effects model.

Results: The pooled prevalence of Shigella species in Ethiopia was $6.6 \%$ (95\% Cl 4.7-8.8). In the subgroup analysis, the highest prevalence was observed among patients in Health facility $(8.5 \%, 95 \% \mathrm{Cl} 6.2-11.5)$ whereas the lowest prevalence was observed in Community based studies (1.6\%, 95\% Cl 0.8-3.4). In addition, Shigella species were highly resistant to ampicillin, amoxicillin, erythromycin and multi-drug resistant (MDR) with the pooled resistance proportions of $83.1 \%$ (95\% Cl 75.7-88.6), 84.1\% (95\% Cl 75.6-90.1), 86.5\% (95\% Cl 70.9-94.4) and 83.2\% (95\% Cl 77.1-87.9), respectively. On the other hand, comparably low resistance pattern was reported for ciprofloxacin $8.9 \%(95 \% \mathrm{Cl}$ 6.0-12.8), ceftriaxone 9.3\% (95\% Cl 3.9-20.5), and norfloxacin 8.2\% (95\% Cl 3.8-16.6) and gentamycin 17.3\% (95\% Cl 11.2-25.9). Subgroup analyses indicated that study years were associated with a decreasing Shigella prevalence over time $(p=0.002)$.

Conclusion: The pooled estimate showed high burden of Shigella infection and its high proportion of drug resistance pattern to ampicillin, amoxicillin and erythromycin in Ethiopia. Therefore, initiating and scale up of performing drug susceptibility test for each shigellosis case, educate the community and health care providers on appropriate use of antibiotics need to be considered and strengthened.
\end{abstract}

Keywords: Systematic review, Meta-analysis, Prevalence, Shigella species, Resistance, Ethiopia

\section{Background}

Shigella species are a major cause of dysentery disease and may attribute for death worldwide. Shigellosis is a major public health threat in developing countries like Ethiopia, people living with poor sanitation and overcrowded condition. Annual number of Shigellosis

\footnotetext{
*Correspondence: sirajhu123@gmail.com

${ }^{1}$ Department of Medical Laboratory Science, College of Medicine and Health Sciences, Hawassa University, Hawassa, Ethiopia Full list of author information is available at the end of the article
}

episodes globally estimated to be about 165 million, more than half (100 million) of episodes occurs in low and middle income countries, and attributed for more than 1 million deaths per year. Shigellosis is more prevalent among under five children, and causes dysentery and under five mortality $[1,2]$.

Antibiotic resistance becomes a critical public health problem around the globe in recent years. Based on 2016 WHO report, Shigella species is one of the eight dangerous drug resistance bacteria. Worldwide, there are 700,000 deaths as a result of antimicrobial resistance 
(AMR) every year. The experts suggest that this figure will rise to 4.2 million in Africa and 10 million globally by 2050, if nothing is done [3-5].

The treatment of Shigellosis has currently become more challenging due to the emergence of drug resistant species and associated with a variety of biological, pharmacological and societal variables with the worst combinations in low and middle income countries [6-8]. Multidrug-resistant Shigella significantly vary from area to area of the world in relation with the practice of widespread use of antimicrobial agents $[3,9]$.

Antibiotic resistance is a natural phenomenon that occurs whenever antibiotics are in use. However, there are human behaviors that contribute to the rapid development and spread of bacterial antibiotic resistance. Availability and use of broad spectrum antibiotic without prescriptions facilitate the development of resistance by Shigella species [10].

Different studies have been carried out in different parts of Ethiopia at different times to document the epidemiology of and drug Susceptibility pattern of Shigella species. However, there is no summarized prevalence data of this bacterial infection and its drug Susceptibility pattern at country level to help in the formulation of appropriate intervention methods. Therefore, the present study is the first of its kind and aimed to determine the pooled prevalence pooled prevalence and drug susceptibility pattern of Shigella species in Ethiopia.

\section{Methods}

\section{Search strategy}

A comprehensive literature search was conducted on the prevalence of shigella species and its antimicrobial resistance pattern among Ethiopia population. Potentially relevant studies were identified through a literature search of Medline, PubMed, Google scholar, HINARI and Cochrane Library. The search was based on the combination of the following special index search terms (medical subject headings (MeSH) and Boolean operations: "Shigella" AND "Prevalence "OR "Epidemiology" AND "Drug Resistance, Microbial” AND "Dysentery, Bacillary/ epidemiology" AND "Ethiopia" "title and abstract" from January 1st 1999 to November 20th 2018. Articles search were focused on published studies with epidemiological and/or clinical data. All records were managed in Endnote version X7 (Clarivate Analytics, Philadelphia, PA, USA). The search was carried out from April 5th, 2016 to November 20, 2018. The limit of language was English and the limit of study group was human.

\section{Eligibility criteria}

We reviewed abstracts from initial search using defined inclusion and exclusion criteria.
Inclusion criteria Studies were selected for systematic review and meta-analysis, 1 , if they were conducted in Ethiopia 2, study design been cross-sectional 3, studies reported the prevalence of Shigella species and its drug resistance pattern 4, literatures published in the English language and 5. Published articles were considered.

Exclusion criteria Studies, which were not fully accessed after reading the titles and abstracts were excluded since we are unable to assess the quality of each article in the absence of their full texts.

\section{Data extraction}

The data extraction was done by three researchers (S.H, Z.Y and G.M) independently from included studies using a standardized and pretested format prepared in Microsoft Excel. The data abstraction format included first author, study design, region in the country (study site in the country), publication year, sample size, population characteristics, age group of study participants, prevalence of Shigella species, medical treatment type, and resistance pattern of Shigella species. Disagreement on data extractions between researchers were resolved through discussion and consensus.

\section{Quality assessment}

The quality was assessed using 9 point Joanna Briggs Institute (JBI) critical appraisal tools, the following criteria is established: sample frame appropriate to address the target population, study participants sampled in an appropriate way, adequate sample size, study participants sampled in an appropriate way, study subjects and the setting described in detail, data analysis conducted with sufficient coverage of the identified sample, valid methods used for the identification of the condition, the condition measured in a standard and reliable way for all participants, appropriate statistical analysis and adequate response rate. Individual studies were assigned a score that was computed using different parameters in line with the review objectives. The responses were scored 0 for "No and not reported" and 1 for "Yes". Total scores ranged between 0 and 9. Studies with medium (fulfilling $50 \%$ of quality assessment parameter) and high quality were included for analysis [11] (Additional file 1: Table S1).

\section{Statistical analysis}

The data entry and analysis were done using Comprehensive Meta-Analysis (version 3.1) software. The original articles were described using forest plot, figures and tables. Since there was heterogeneity among studies, random effect model was used to compute the pooled prevalence and antimicrobial susceptibility of Shigella species. 
The estimated pooled prevalence rate with $95 \%$ confidence interval (CI) was presented.

\section{Sub-group analysis}

Sub-group analysis was performed based on Region; (Amhara, Oromia Southern Ethiopia, Central Ethiopia, Hareri and Tigray), age group; (children, adult and all age group), study population (health facility based and community based) and year of study; (1999-2003, 20042008, 2009-2013 and 2014-2018).

\section{Heterogeneity and publication bias}

Statistical heterogeneity was evaluated by Cochran's Q test and $\mathrm{I}^{2}$ statistic. The $\mathrm{I}^{2}$ provides an estimate of the percentage of the variability in effect estimates that is due to heterogeneity rather than sampling error or chance differences. So, the existence of heterogeneity was verified using Cochran's $Q$ test $(\mathrm{p}<0.10$ indicates statistically significant heterogeneity) and $\mathrm{I}^{2}$ test that measures level of statistical heterogeneity between studies. $\mathrm{I}^{2}$ (values of $25 \%, 50 \%$ and $75 \%$ are considered to represent low, medium and high heterogeneity respectively) [12, 13]. Begg intercept and Mazumdar rank correlation statistics test methods were used to statistically assess publication bias ( $\mathrm{p}<0.05$ was consider as suggestive of statistically significant publication bias) [14]. As the results of the test suggested a possible existence of a significant publication bias, the final effect size was determined by applying Duval and Tweedie's Trim and Fill analysis in the random-effects model.

\section{Results}

\section{Identified studies}

Through electronic database search, we have found a total of 372 studies. Of which, 335 were excluded based on the titles and abstracts, three article were not reported prevalence of Shigella species and the remaining five were excluded due to unavailability of full text articles. Finally, 29 studies were found to be eligible and included in the meta-analysis (Fig. 1). Included articles exhibited high heterogeneity according to Cochrane $\mathrm{Q}$ test $(\mathrm{Q}=437.836$ test $\mathrm{p}<0.0001)$ and $\mathrm{I}^{2}$ test $\left(\mathrm{I}^{2}=93.605 \%\right)$, which is indicative to use random effects model. The distribution of the studies using funnel plot (Fig. 2) showed asymmetrical distribution of effect estimate and Eggers regression intercept test $(\mathrm{p}<0.0001)$ and Begg and Mazumdar rank correlation $(p<0.001)$ indicated evidence of publication bias (Additional file 2: Figures S1 and S2). Therefore, we were used Trim and Fill analysis to adjust the final pooled estimate.

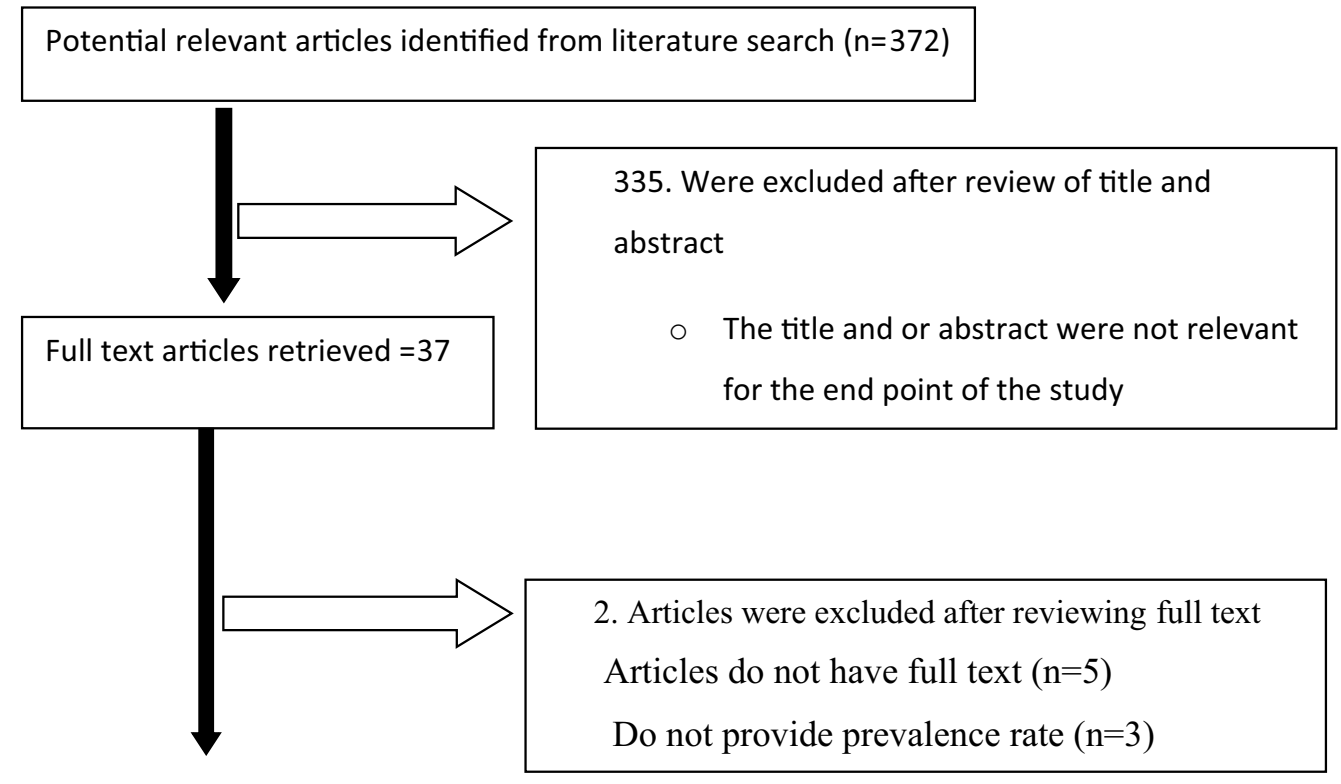

Studies Included in systematic review and meta-analysis $=29$

Fig. 1 Flow diagram of studies reviewed, screened and included 


\section{Funnel Plot of Standard Error by Logit event rate}

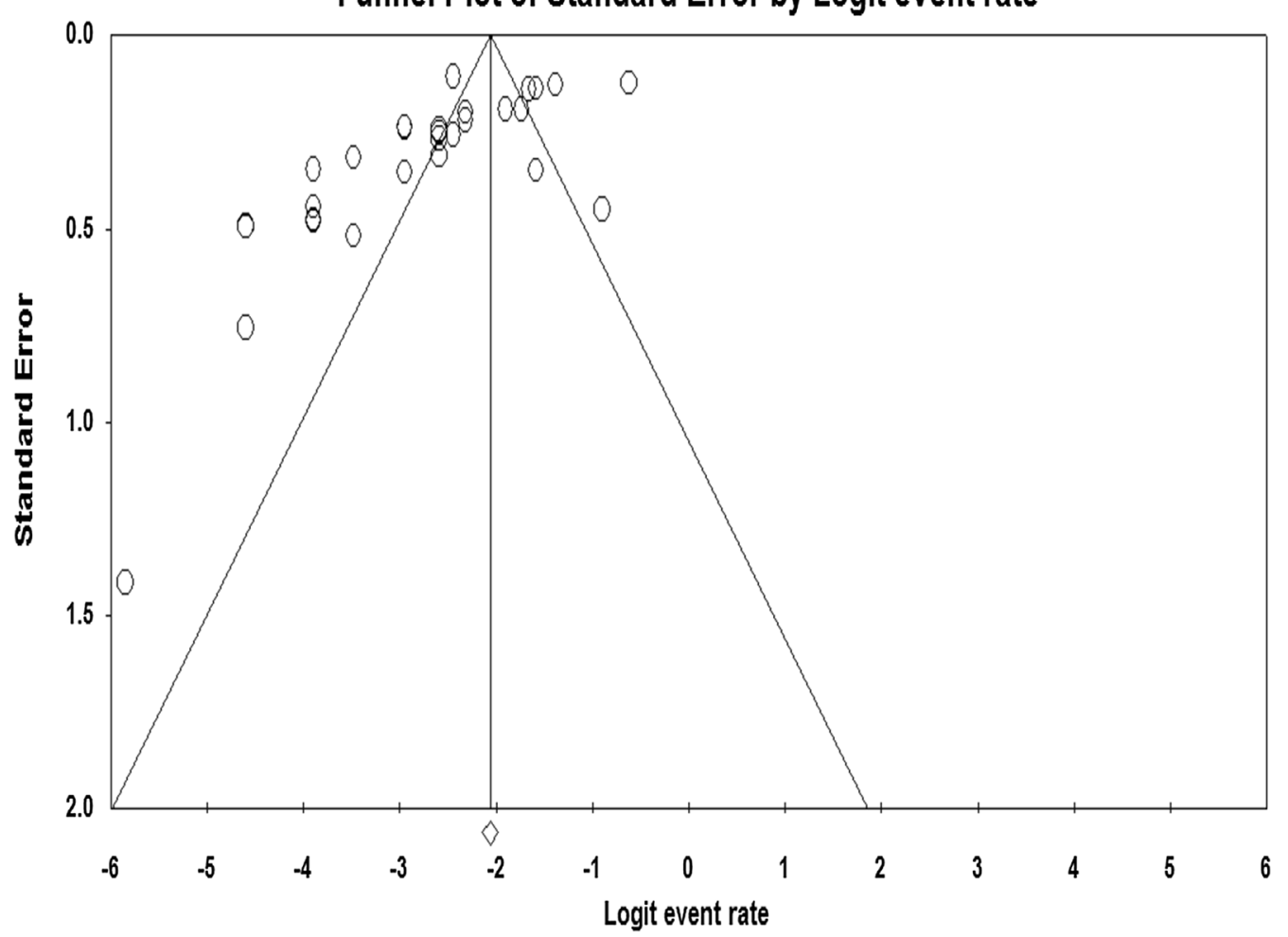

Fig. 2 Funnel plot for the prevalence of Shigella species in Ethiopia

\section{Study characteristics}

Selected articles were published from 1999 to 2018. Also, all included publications were obtained from 5 regions and 1 City administration, but no data was obtained from other regions (Afar, BenishangulGumuz, Gambela and Somali). The total study populations involved for the estimation of Shigella species were (8521). Of this, 7571 study participants were included in the meta-analysis of Shigella species drug resistant pattern. A total of twenty-nine studies were considered eligible for quantitative syntheses. Twentyfive studies were used to estimate the proportions of drug resistant for Shigella species (Table 2). Nine studies were used to estimate the prevalence of Shigella species in children and seven studies were used to estimate for adult population. Of the total studies, only seven reported the serogroup of Shigella (Table 1). Among study populations, 3907 (45.85\%) were examined for Shigella and its antimicrobial resistance from Amhara region. About 1683 (19.75\%) from Oromia region, 1545 (18.13\%) from southern region, 425 (4.99\%) from Central Ethiopia (Addis Ababa), 244 (2.86\%) from Harare region and 717 (8.41\%) were from Tigray region. Cross sectional study design was used in all studies. The study population varied from 24 to 1200 , and were conducted between the years 1999-2018 [15-43] (Table 1). All studies utilized stool specimens for diagnosis of Shigella species (Table 1). Data from health facility based study: 6717 patients with diarrhea and 113 without diarrhea and from community based study: 1691 apparently healthy subjects were considered for quantitative syntheses. The prevalence of Shigella in stool samples of apparently healthy subjects and patients ranged from 0.0 to $3.1 \%$ from 1.1 to $34.6 \%$, respectively. MacConkey, xylose lysine desoxycholate and Salmonella Shigella agar used for culture and disk diffusion method for antimicrobial susptablity (Additional file 1: Table S2).

\section{Meta-analysis of Shigella species}

The analysis of 29 studies, according to the Der Simonian-Laird random-effects model, revealed that the pooled prevalence of Shigella species in Ethiopia was 6.6\% (95\% CI 4.7-8.8) (Fig. 3). Pooled prevalence of Shigella species among regions was $7.0 \%$ (95\% CI 4.6-10.3) in Amhara, 4.1\% (95\% CI 1.0-14.5) in Oromia, $7.7 \%$ (95\% CI 2.7-20.1) in Southern Ethiopia, 2.2\% (95\% CI 0.1-40.5) in Addis Ababa, 7.0\% (95\% CI 4.4-11.0) in Hareri and $8.8 \%$ (95\% CI 5.6-13.6) in Tigray. In year of study $26.9 \%$ (95\% CI 14.8-43.8) in 1999-2003, $12.6 \%$ (95\% CI 6.6-22.7) in 2004-2008, 8.1\% (95\% CI 5.1-12.8) 


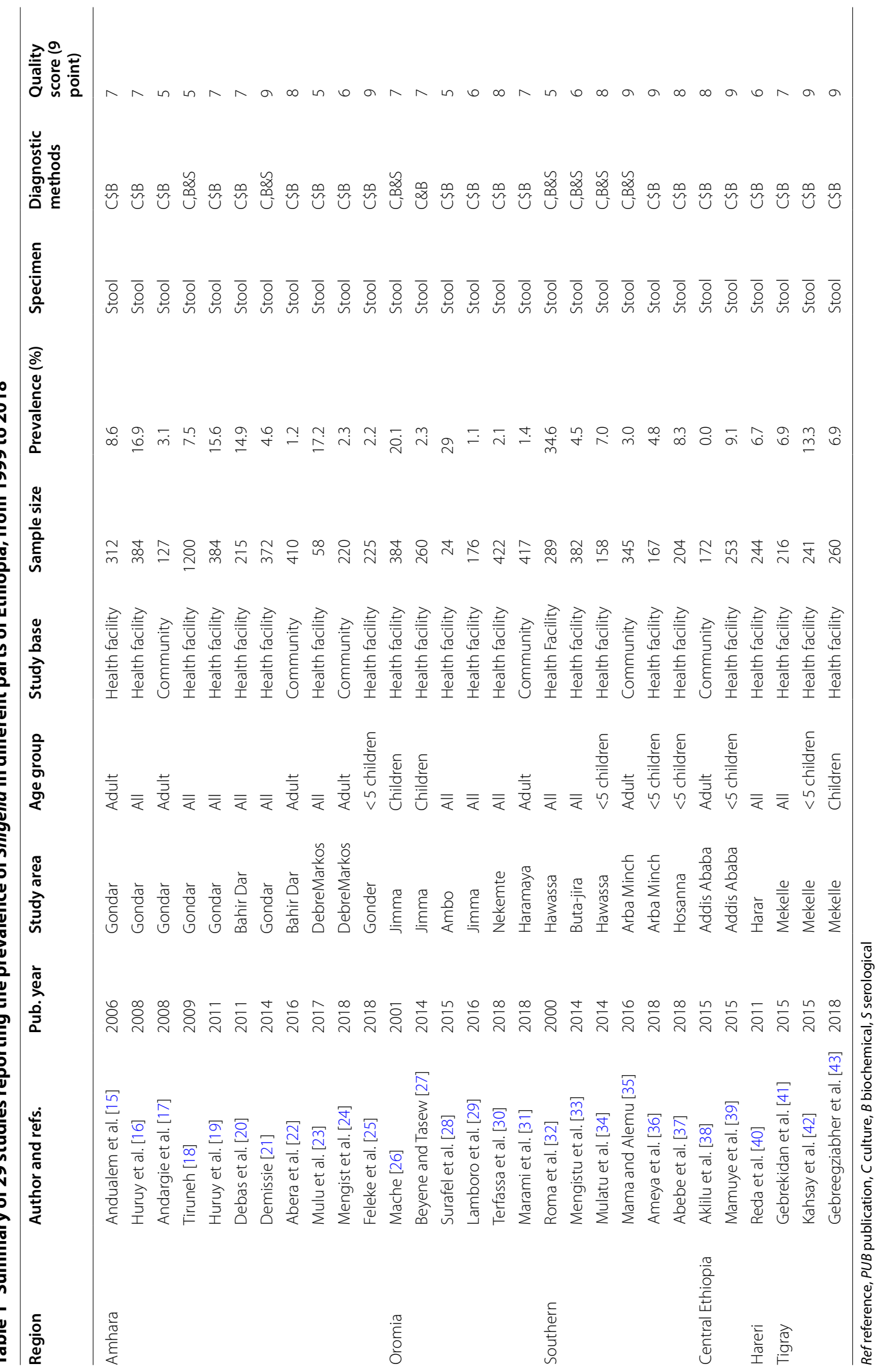




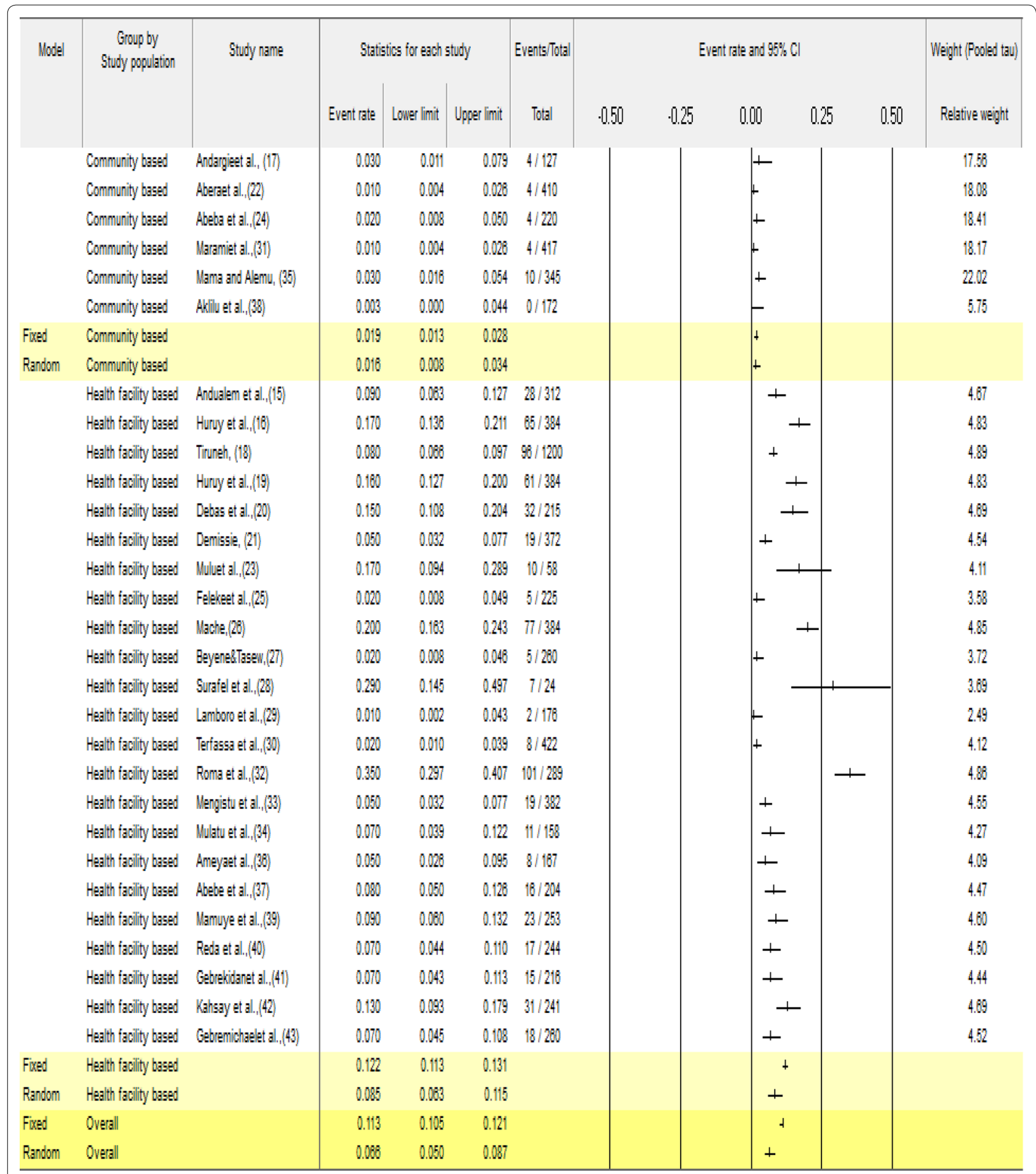

Fig. 3 Forest plot for the prevalence of Shigella species in Ethiopia

in 2009-2013 and 4.9\% (95\% CI 3.3-7.0) from 2014 to 2018 (Additional file 1: Table S2) and among study population 1.8 (95\% CI 1.1-2.9) were in health facility based and $1.6 \%$ (95\% CI $0.8-3.4$ ) in Community based studies.
Among the above study parameters considered during meta-regression analysis, study years were associated with a decreasing Shigella prevalence over time $(\mathrm{p}=0.0028)$. 


\section{Meta-analysis of drug resistant Shigella species}

The pooled resistant of Shigella species were 83.1\% (95\% CI 75.7-88.6) for ampicillin, 84.1\% (95\% CI 75.6-90.1) for Amoxicillin and 86.5\% (95\% CI 70.9-94.4) for erythromycin. Comparatively low resistance pattern was reported in ciprofloxacin $8.9 \%$ (95\% CI 6.0-12.8), ceftriaxone 9.3\% (95\% CI 3.9-20.5), gentamycin $17.3 \%$ (95\% CI 11.225.9), and norfloxacin $8.2 \%$ (95\% CI 3.8-16.6). However intermediate resistance were recorded for augmentin (Amox + clav) 59.1\% (95\% CI 19.6-89.5) and co-trimoxazele 59.4\% (95\% CI 49.3-68.8). Moreover, resistant to more than one drugs or MDR were reported $83.2 \%$ (95\% CI 77.1-87.9) (Table 2). Above 50\% of Shigella developed resistance to ampicillin, erythromycin, tetracycline, cotrimoxazole and chloramphenicol which are the commonly prescribed antimicrobial drugs. Most studies on drug resistance were carried out in 2014-2018, though it is difficult to sufficiently address and compare the resistance pattern of commonly prescribed drugs through time (Additional file 1: Table S4).

\section{Discussion}

Antimicrobial resistance (AMR) is widely growing public health threat worldwide, particularly in resource limited countries including Ethiopia, where infectious diseases are widespread. It is now occurring across the world [1].

Shigella species is highly antibiotic resistant among eight drugs resistance bacteria. Regarding antibiotic resistance, if nothing is done, a person will die every three second by 2050 [44].

This meta-analysis determined the pooled prevalence of shigella species in Ethiopia using 29 studies. According to the results of this meta-analysis, the pooled prevalence was $6.6 \%$ (95\% CI 4.7-8.8). This finding is in agreement with $6.6 \%$ (95\% CI 3.4-9.7) Shegilla prevalence in systematic review among US military and similar populations [45].

Regional prevalence of Shigella species among regions was also calculated, hence a higher prevalence of Shigella species (8.8\%) was reported in Tigray, which was nearly four times higher than the finding from Central Ethiopia (2.2\%), even though the studies conducted and included in this review and meta-analysis from this region were small. The variations in prevalence estimates may be due to differences in the study populations and year of study.

Pooled prevalence of Shigella species by study year (26.9\%) was reported in 1999.2003 and (4.9\%) in 20142018. This finding is inconsistent with the study done in China [46]. This result showed a decreasing Shigella prevalence over time. The decreased in prevalence through time might be due to decrease in poverty, increase quality of life, rise of awareness on sanitation and hygiene, prevention and control strategy of communicable disease through deployed of health extension workers at community level across the country.

In this study, the pooled prevalence of shigellosis in children was $(7.0 \%)$, while in adult population (2.2\%). This finding confirms that Shigella cause diarrhoeal morbidity among infants and young children than adults [47] and the third leading cause of diarrhoeal deaths in children younger than 5 years [48]. The higher occurrence of Shigella in children compared to adults suggests a higher vulnerability of children to Shigella infection, this may be due to the unhygienic food handling practices, compromised sanitation, malnutrition and the ability to cause disease with a small inoculum and facilitating spread from person to person [49].

Based on the data obtained from twenty-five published articles; Shigella species showed high resistance to amoxicillin, ampicillin, and erythromycin. This finding is in line with the study done in Germany [50].

In Ethiopia the drug of choice on shigellosis treatment is norfloxacin, ciprofloxacin, and ceftriaxone for adult. This is similar with guideline USA [51]. However this review and meta-analysis showed slightly high resistance was reported on norfloxacin, ciprofloxacin, and ceftriaxone.

Furthermore, the occurrences of Shigella isolates resistant to two or more drugs were high (83.2\%). This increment may be due mobile genetic units (including plasmids, gene cassettes in integrons and transposons) [1], inadequate access to effective drugs, unregulated dispensing, truncated antimicrobial therapy, medication sharing, counterfeit drugs, bacterial evolution, climate changes, lack of medical practitioner with proper training, poor-quality and unhygienic sanitary conditions [52-54].

In this review, included studies primarily used stool culture for Shigella identification. This estimate appears to be less sensitive method than molecular methods and may underestimate the actual occurrence of this bacteria [47].

\section{Conclusion}

The pooled estimate provides high burden of Shigella infection and its high proportion of drug resistance pattern to ampicillin, Amoxicillin and Erythromycin in Ethiopia. Therefore, initiating and scale up of performing drug susceptibility test for each shigellosis case, educate the community and health care providers on appropriate use of antibiotics need to be considered and strengthened. 


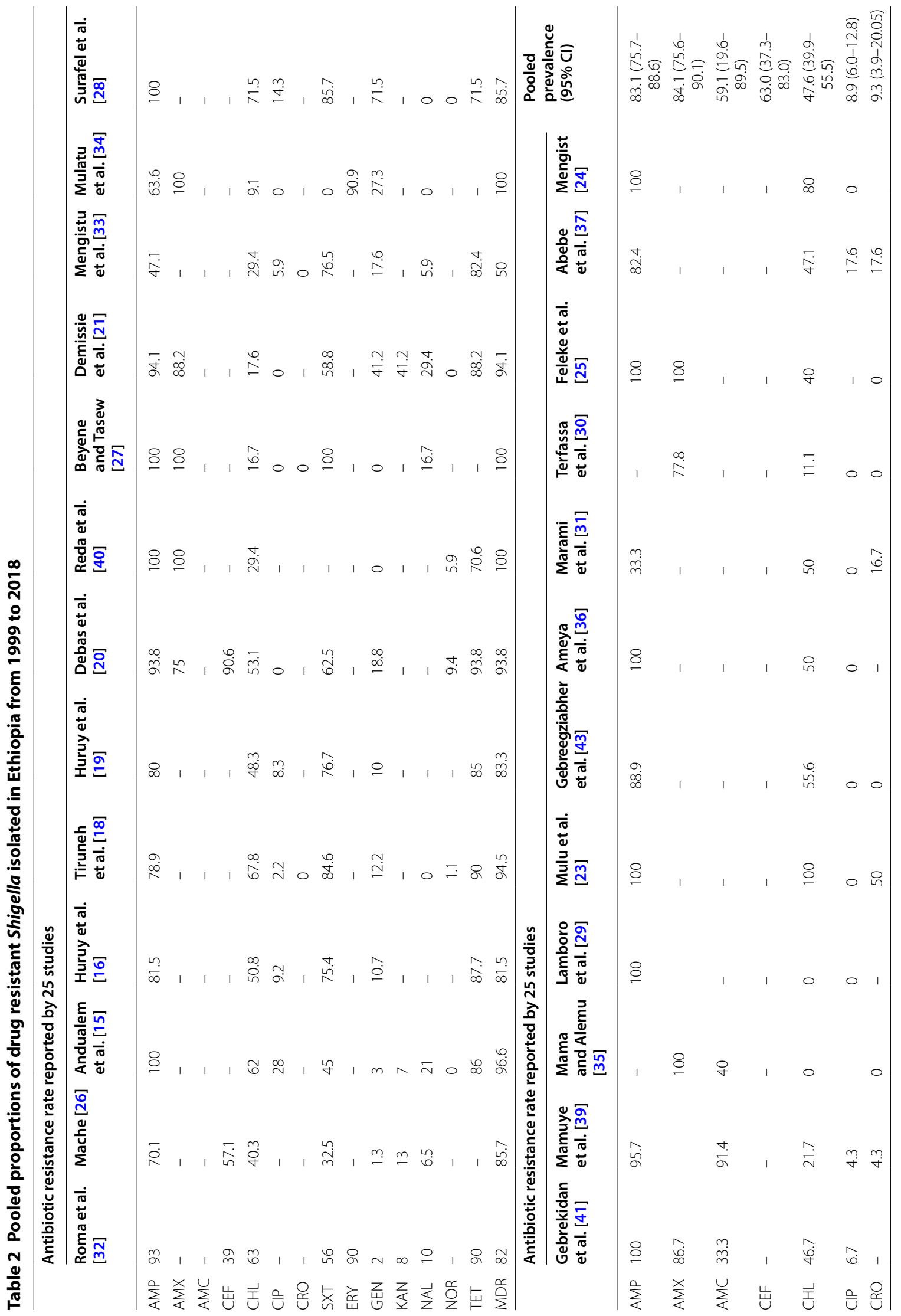




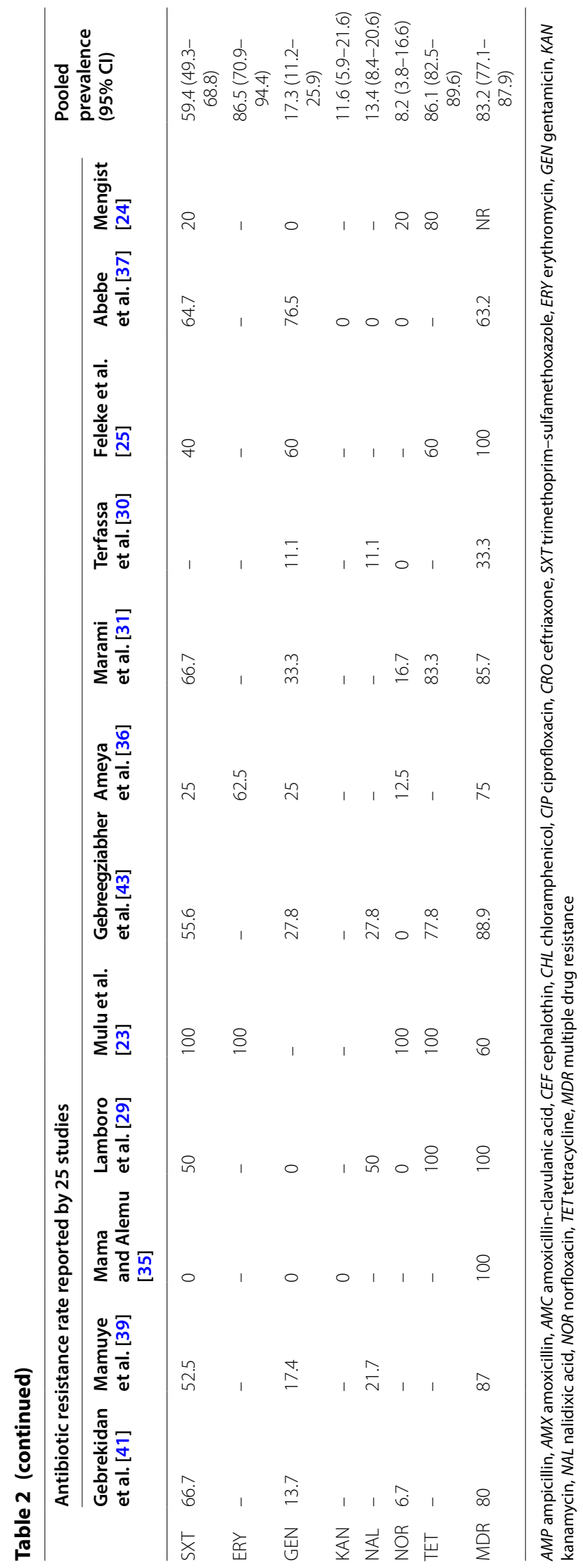




\section{Limitation}

The patient data were collected from urban and rural populations of the country that took service from hospitals but urban dwellers have better access to health care setting. Therefore, the pooled estimates are more applicable to patients in urban areas than rural. Resistance pattern of shigellosis was not reported for money drugs by some studies and in most studies Shigella infection were not tested at species level.

\section{Additional files}

Additional file 1: Table S1. Study design and quality assessment of the studies included in systematic review and meta-analysis of shigella species. Table S2. Subgroup meta-analysis of Shigella species prevalence estimation in Ethiopia from 1999 to 2018. Table S3: Microbiological methods used to isolate and identify Shigella species in Ethiopia from 1999 to 2018. Table S4. Prevalence of drug resistance $(95 \%$ Cl) for Shigella from 1999 to 2018.

Additional file 2: Figure S1. Egger regression intercept of analysis of included studies reporting on the prevalence of Shigella species in Ethiopia. Figure S2. Begg and Mazumdar rank correlation of analysis of included studies reporting on the prevalence of Shigella species in Ethiopia.

\section{Abbreviations}

AMR: antimicrobial resistance; MDR: multi-drug resistant.

\section{Acknowledgements}

We don't have any person or organization to acknowledge.

\section{Authors' contributions}

$\mathrm{SH}$ was the principal investigator who contributed to origin, the idea and design of the study, collected, entered, analyzed, interpreted the data, prepared the manuscript and acted as corresponding author. GM and ZYK contributed to data analysis, interpretation and drafted the manuscript. All authors read and approved the final manuscript.

\section{Funding}

There was no any funding or sponsoring organization for this paper

\section{Availability of data and materials}

There is no remaining data and materials, all information is clearly presented in the main manuscript.

\section{Ethics approval and consent to participate}

Not applicable.

\section{Consent for publication}

This manuscript does not contain data from any other person's. So not applicable.

\section{Competing interests}

The authors declare that they have no competing interests.

\section{Author details}

1 Department of Medical Laboratory Science, College of Medicine and Health Sciences, Hawassa University, Hawassa, Ethiopia. ${ }^{2}$ School of Nursing and Midwifery, College of Medicine and Health Sciences, Hawassa University, Hawassa, Ethiopia.
Received: 1 April 2019 Accepted: 29 June 2019

Published online: 09 July 2019

\section{References}

1. World Health Organization. Antimicrobial resistance: global report on surveillance. Geneva: World Health Organization; 2014.

2. Temu M, Kaatano G, Miyaye N, Buhalata S, Shushu M, Kishamawe C, et al. Antimicrobial susceptibility of Shigella flexneri and S. dysenteriae isolated from stool specimens of patients with bloody diarrhoea in Mwansa, Tanzania. Tanzan J Health Res. 2007;9(3):186-9.

3. World Health Organization. The world health report 2007: a safer future: global public health security in the 21st century. Geneva: World Health Organization; 2007

4. Woolhouse M, Waugh C, Perry MR, Nair H. Global disease burden due to antibiotic resistance-state of the evidence. J Glob Health. 2016;6(1):010306

5. Lima AÂM. Tropical diarrhoea: new developments in traveller's diarrhoea. Curr Opin Infect Dis. 2001;14(5):547-52.

6. Porter C, Thura N, Ranallo R, Riddle M. The Shigella human challenge model. Epidemiol Infect. 2013;141(2):223-32.

7. Farthing M, Lindberg G, Dite P, Khalif I, Salazar-Lindo E, Ramakrishna B, et al. World Gastroenterology Organisation practice guideline: acute diarrhea. Milwaukee: World Gastroenterology Organisation; 2008. p. 7-12.

8. Al-Nimri S, Miller WA, Byrne BA, Guibert G, Chen L. A unified approach to molecular epidemiology investigations: tools and patterns in California as a case study for endemic shigellosis. BMC Infect Dis. 2009;9(1):184.

9. Čižman M. The use and resistance to antibiotics in the community. Int J Antimicrob Agents. 2003;21(4):297-307.

10. UNICEF/WHO. Diarrhoea: why children are still dying and what can be done. Geneva: UNICEF/WHO; 2009.

11. JBI critical appraisal checklist for studies reporting prevalence data; 2016.

12. DerSimonian R, Laird N. Meta-analysis in clinical trials. Control Clin Trials. 1986;7(3):177-88.

13. Rücker G, Schwarzer G, Carpenter JR, Schumacher M. Undue reliance on 12 in assessing heterogeneity may mislead. BMC Med Res Methodol. 2008;8(1):79.

14. Ioannidis J. Interpretation of tests of heterogeneity and bias in metaanalysis. J Eval Clin Pract. 2008;14(5):951-7.

15. Andualem B, Kassu A, Diro E, Moges F, Gedefaw M. The prevalence and antimicrobial responses of Shigella isolates in HIV-1 infected and uninfected adult diarrhoea patients in North West Ethiopia. Ethiop J Health Dev. 2006:20(2):99-105.

16. Huruy K, Kassu A, Mulu A, Gebretsadik S, Andargie G, Tadesse T, et al. High level of antimicrobial resistance in Shigella species isolated from diarrhoeal patients in University of Gondar Teaching Hospital, Gondar, Ethiopia. Pharmacol Online. 2008;2:328-40.

17. Andargie G, Kassu A, Moges F, Tiruneh M, Huruy K. Prevalence of bacteria and intestinal parasites among food-handlers in Gondar town, northwest Ethiopia. J Health Popul Nutr. 2008;26(4):451.

18. Tiruneh M. Serodiversity and antimicrobial resistance pattern of Shigella isolates at Gondar University teaching hospital, Northwest Ethiopia. Jpn J Infect Dis. 2009;62(2):93-7.

19. Huruy K, Kassu A, Mulu A, Worku N, Fetene T, Gebretsadik S, Biadglegne F, Belyhun Y, Muche A, Gelaw A, Anagaw B, Yifru S, Wondie Y, Bekele A, Tiruneh M, Reissig D, Moges F. Intestinal parasitosis and shigellosis among diarrheal patients in Gondar teaching hospital, northwest Ethiopia. BMC Res Notes. 2011;4:472. https://doi.org/10.1186/1756-0500-4-472.

20. Debas G, Kibret M, Biadglegne F, Abera B. Prevalence and antimicrobial susceptibility patterns of Shigella species at Felege Hiwot Referral Hospital, Northwest Ethiopia. Ethiop Med J. 2011;49(3):249-56.

21. Demissie A, Wubie T, Yehuala FM, Fetene M, Gudeta A. Prevalence and antimicrobial susceptibility patterns of Shigella and Salmonella species among patients with diarrhea attending Gondar Town Health Institutions, Northwest Ethiopia. Sci J Pub Health. 2014;2(5):469-75.

22. Abera B, Yitayew G, Amare H. Salmonella serotype Typhi, Shigella, and intestinal parasites among food handlers at Bahir Dar University, Ethiopia. J Infect Dev Ctries. 2016;10(2):121-6. https://doi.org/10.3855/jidc.6890. 
23. Mulu W, Abera B, Yimer M, Hailu T, Ayele H, Abate D. Bacterial agents and antibiotic resistance profiles of infections from different sites that occurred among patients at Debre Markos Referral Hospital, Ethiopia: a cross-sectional study. BMC Res Notes. 2017;10(1):254. https://doi. org/10.1186/s13104-017-2584-y.

24. Mengist A, Mengistu G, Reta A. Prevalence and antimicrobial susceptibility pattern of Salmonella and Shigella among food handlers in catering establishments at Debre Markos University, Northwest Ethiopia. Int J Infect Dis. 2018:75:74-9.

25. Feleke H, Medhin G, Abebe A, Beyene B, Kloos H, Asrat D. Enteric pathogens and associated risk factors among under-five children with and without diarrhea in Wegera District, Northwestern Ethiopia. Pan Afr Med J. 2018;29:1

26. Mache A. Antibiotic resistance and sero-groups of Shigella among paediatric out-patients in southern Ethiopia. East Afr Med J. 2001;78(6):296-9.

27. Beyene G, Tasew H. Prevalence of intestinal parasite, Shigella and Salmonella species among diarrheal children in Jimma health center, Jimma southwest Ethiopia: a cross sectional study. Ann Clin Microbiol Antimicrob. 2014;13(1):10.

28. Kefyalew S, Kebede G, Keneni A. Prevalence of Shigella related diarrhea in Ambo Town and antibiotic susceptibility of the isolated strains. Greener J Epidemiol Public Health. 2015. https://doi.org/10.15580/GJEPH 2015.1.120714400.

29. Lamboro T, Ketema T, Bacha K. Prevalence and antimicrobial resistance in Salmonella and Shigella species isolated from outpatients, Jimma University Specialized Hospital, Southwest Ethiopia. Can J Infect Dis Med Microbiol. 2016;2016:4210760.

30. Terfassa A, Jida M. Prevalence and antibiotics susceptibility pattern of Salmonella and Shigella species among diarrheal patients attend ing Nekemte Referral Hospital, Oromia, Ethiopia. Int J Microbiol. 2018:24(2018):9214689. https://doi.org/10.1155/2018/9214689.

31. Marami D, Hailu K, Tolera M. Prevalence and antimicrobial susceptibility pattern of Salmonella and Shigella species among asymptomatic food handlers working in Haramaya University cafeterias, Eastern Ethiopia. BMC Res Notes. 2018;11(1):74. https://doi.org/10.1186/s1310 4-018-3189-9.

32. Roma B, Worku S, Mariam ST, Langeland N. Antimicrobial susceptibility pattern of Shigella isolates in Awassa. Ethiop J Health Dev. 2000;14(2):149-54.

33. Mengistu G, Mulugeta G, Lema T, Aseffa A. Prevalence and antimicrobial susceptibility patterns of Salmonella serovars and Shigella species. J Microb Biochem Technol. 2014;6(S2):S2-006.

34. Mulatu G, Beyene G, Zeynudin A. Prevalence of Shigella, Salmonella and Cmpylobacter species and their susceptibility patters among under five children with diarrhea in Hawassa Town, South Ethiopia. Ethiop J Health Sci. 2014;24(2):101.

35. Mama M, Alemu G. Prevalence, antimicrobial susceptibility patterns and associated risk factors of Shigella and Salmonella among food handlers in Arba Minch University, South Ethiopia. BMC Infect Dis. 2016;16(1):686.

36. Ameya G, Tsalla T, Getu F, Getu E. Antimicrobial susceptibility pattern, and associated factors of Salmonella and Shigella infections among under five children in Arba Minch, South Ethiopia. Ann Clin Microbiol Antimicrob. 2018;17(1):1. https://doi.org/10.1186/s12941-018-0253-1.

37. Abebe W, Earsido A, Taye S, Assefa M, Eyasu A, Godebo G. Prevalence and antibiotic susceptibility patterns of Shigella and Salmonella among children aged below five years with Diarrhoea attending Nigist Eleni Mohammed memorial hospital, South Ethiopia. BMC Pediatr. 2018;18(1):241.

38. Aklilu A, Kahase D, Dessalegn M, Tarekegn N, Gebremichael S, Zenebe S, et al. Prevalence of intestinal parasites, Salmonella and Shigella among apparently health food handlers of Addis Ababa University student's cafeteria, Addis Ababa, Ethiopia. BMC Res Notes. 2015;8(1):17.

39. Mamuye Y, Metaferia G, Birhanu A, Desta K, Fantaw S. Isolation and antibiotic susceptibility patterns of Shigella and Salmonella among under 5 children with acute diarrhoea: a cross-sectional study at selected public health facilities in Addis Ababa, Ethiopia. Clin Microbiol. 2015. https://doi. org/10.4172/2327-5073.1000186.

40. Reda AA, Seyoum B, Yimam J, Fiseha S, Jean-Michel V. Antibiotic susceptibility patterns of Salmonella and Shigella isolates in Harar, Eastern Ethiopia. J Infect Dis Immun. 2011;3(8):134-9.
41. Gebrekidan A, Dejene TA, Kahsay G, Wasihun AG. Prevalence and antimicrobial susceptibility patterns of Shigella among acute diarrheal outpatients in Mekelle hospital, Northern Ethiopia. BMC Res Notes. 2015;8(1):611.

42. Kahsay AG, Teklemariam Z. Prevalence of Shigella among diarrheic children under-5 years of age attending at Mekelle health center, north Ethiopia. BMC Res Notes. 2015;8(1):788.

43. Gebreegziabher G, Asrat D, Hagos T. Isolation and antimicrobial susceptibility profile of Shigella and Salmonella species from children with acute diarrhoea in Mekelle Hospital and Semen Health Center, Ethiopia. Ethiop J Health Sci. 2018;28(2):197-206.

44. Globally TD-RI. Final report and recommendations. Review on antimicrobial resistance report; 2016.

45. Riddle MS, Sanders JW, Putnam SD, Tribble DR. Incidence, etiology, and impact of diarrhea among long-term travelers (US military and similar populations): a systematic review. Am J Trop Med Hyg. 2006;74(5):891-900.

46. Shane AL, Mody RK, Crump JA, Tarr PI, Steiner TS, Kotloff K, et al. 2017 Infectious diseases society of america clinical practice guidelines for the diagnosis and management of infectious diarrhea. Clin Infect Dis. 2017;65(12):e45-80.

47. Liu J, Platts-Mills JA, Juma J, et al. Use of quantitative molecular diagnostic methods to identify causes of diarrhoea in children: a reanalysis of the GEMS case-control study. Lancet. 2016;388:1291-301.

48. GBD Diarrhoeal Diseases Collaborators. Estimates of global, regional, and national morbidity, mortality, and aetiologies of diarrhoeal diseases: a systematic analysis for the Global Burden of Disease Study 2015. Lancet Infect Dis. 2017;17:909-48.

49. DuPont HL, Levine MM, Hornick RB, Formal SB. Inoculum size in shigellosis and implications for expected mode of transmission. J Infect Dis. 1989;159:1126-8.

50. Visschers VH, Backhans A, Collineau L, Iten D, Loesken S, Postma M, et al. Perceptions of antimicrobial usage, antimicrobial resistance and policy measures to reduce antimicrobial usage in convenient samples of Belgian, French, German, Swedish and Swiss pig farmers. Prev Vet Med. 2015;119(1-2):10-20.

51. Chang Z, Lu S, Chen L, Jin Q, Yang J. Causative species and serotypes of shigellosis in mainland China: systematic review and meta-analysis. PLoS One. 2012;7(12):e52515.

52. Fewtrell L, Kaufmann RB, Kay D, Enanoria W, Haller L, Colford JM. Water, sanitation, and hygiene interventions to reduce diarrhoea in less developed countries: a systematic review and meta-analysis. Lancet Infect Dis. 2005;5(1):42-52.

53. Putnam S, Riddle M, Wierzba T, Pittner B, Elyazeed R, El-Gendy A, et al. Antimicrobial susceptibility trends among Escherichia coli and Shigella spp. isolated from rural Egyptian paediatric populations with diarrhoea between 1995 and 2000. Clin Microbiol Infect. 2004;10(9):804-10.

54. Church DL. Major factors affecting the emergence and re-emergence of infectious diseases. Clin Lab Med. 2004;24(3):559-86.

\section{Publisher's Note}

Springer Nature remains neutral with regard to jurisdictional claims in published maps and institutional affiliations.

Ready to submit your research? Choose BMC and benefit from:

- fast, convenient online submission

- thorough peer review by experienced researchers in your field

- rapid publication on acceptance

- support for research data, including large and complex data types

- gold Open Access which fosters wider collaboration and increased citations

- maximum visibility for your research: over $100 \mathrm{M}$ website views per year

At BMC, research is always in progress.

Learn more biomedcentral.com/submissions 